\title{
Laparoscopic Ventral Hernia Repair: Tow Centre Experience Prospective Comparative Study
}

\author{
Mohamed Yousef A. ${ }^{*}$, Mohamed Abdel Shafy Mohamed ${ }^{1}$, Asmaa Gaber R. ${ }^{1}$, \\ Mahmoud Salah Ahmed ${ }^{2}$, Hamdy M. Husein'1 \\ ${ }^{1}$ Department of General Surgery, Faculty of Medicine, South Valley University, Qena, Egypt \\ ${ }^{2}$ Department of General Surgery, Faculty of Medicine, Aswan University, Aswan, Egypt \\ Email: *myousef76@gmail.com
}

How to cite this paper: Mohamed Yousef A., Mohamed, M.A.S., Asmaa Gaber R., Ahmed, M.S. and Husein, H.M. (2018) Laparoscopic Ventral Hernia Repair: Tow Centre Experience Prospective Comparative Study. Open Access Library Journal, 5: e4871.

https://doi.org/10.4236/oalib.1104871

Received: August 29, 2018

Accepted: September 16, 2018

Published: September 19, 2018

Copyright $\odot 2018$ by authors and Open Access Library Inc.

This work is licensed under the Creative Commons Attribution International License (CC BY 4.0).

http://creativecommons.org/licenses/by/4.0/

\begin{abstract}
Background: Ventral hernia is one of the most common abdominal wall hernias. Several procedures have been used for hernia repair. During the last few decades, the open surgical approach has been the standard technique for hernia repair. During the past 10 years, laparoscopic repair of ventral hernia has become increasingly established in clinical practice and aimed to be an acceptable and successful technique. There are many techniques used in laparoscopic ventral hernia repair and the most commonly used is fixation of mesh without closing the defect or closing the defect before fixation of mesh. Aim of the Study: The aim of this study is to compare outcomes and results of closure versus non-closure of ventral hernia defect during laparoscopic ventral hernia repair in tow center and report our experience in laparoscopic ventral hernia repair. Patients and Methods: This is comparative prospective study between laparoscopic ventral hernia repair without closure of the defect and with closure of the defect before fixation of the mesh. 60 patients were divided into 2 groups: Group 1 treated with laparoscopic ventral hernia repair without defect closure and group 2 treated with laparoscopic ventral hernia repair with hernia defect closure, and we followed up the patients in both groups for operative outcomes and post-operative complications, hospital stay, recurrences, patients' satisfactions. Results: Operative time was longer in group 2-closure group than in group 1-non-closure group. Post operative seroma is $65 \%$ in group 1 and $16 \%$ in group 2 . Recurrence occurs in one patient [3.33\%] in group 2 versus 4 patients [13.33\%] in group 1. Conclusion: Laparoscopic ventral hernia repair is safe and feasible, although laparoscopic ventral hernia repair without closure of the defect is easy with less operative time and does not need extra-experience in intra-corporeal suturing
\end{abstract}


but its benefit was in smaller defect $[3 \mathrm{~cm}]$ and larger defect needs a laparoscopic ventral hernia repair with defect closure.

\section{Subject Areas}

Surgery \& Surgical Specialties

\section{Keywords}

Laparoscopic Ventral Hernia Repair, With, Without Defect Closure

\section{Introduction}

Ventral hernia is a most common type of abdominal wall hernias and it is considered one of the most common problems that face the general surgeons, with overall incidence between $2 \%$ and $13 \%$ [1] [2] [3] [4]. It occurs either as a complication of previous surgery or occurs naturally without previous operation [1] [2].

Ventral hernia includes all the hernias occurring through the anterior abdominal wall except groin hernias. It includes many types as incisional, para-umbilical, umbilical, parastomal, epigastric \& spigelian [3] [4].

There are numerous risk factors for increasing rates of ventral hernia as general condition of patients [age, sex, generalized wasting, malignant disease, anemia, jaundice, diabetes mellitus, chronic liver failure, ascites, chronic renal failure, prolonged steroid therapy, immunosuppressive therapy and alcoholism] [5]. Also malnutrition, obesity, smoking and collagen disease are playing an important role in incidence of ventral hernia [6] [7]. Also there are many factors related to surgical technique as types of incision; for examples midline vertical incisions have a tendency to burst, which is higher than those, which are transverse [8] and type of closure can play a role as predisposing factor for ventral hernia incidence; layered closures may be followed by higher incidence of post operative hernias than wounds closed by single layer [mass closure technique]. Other important factors are suture material and suturing technique, postoperative complications as seroma and wound infection [8] [9].

There are many investigations of ventral hernia as abdominal US, which is a helpful diagnostic tool, especially in small or barely palpable hernias, or in obese patients, has many advantages as it is non-invasive, time and cost-saving, readily repeatable, and practically risk-free, also abdominal us helpful in detection of location and size of ventral hernia, and allows the determination of hernia content, as well as excluding important differential diagnoses such as lymphoma or hematoma. Computerized Tomography [CT] of the abdominal wall is an excellent method for evaluating the abdominal wall and its relations to the abdominal viscera as lesions can be easily identified and it is the modality of choice in obese patients. Magnetic Resonance Imaging [MRI] is comparable to CT, which offers 
the advantage of direct multiplane imaging without ionizing radiation and the use of contrast agents. A relative merit of MRI is the excellent demonstration of abdominal wall layers [10].

Several procedures have been used for hernia repair. During the last few decades, the open surgical approach has been the standard technique for hernia repair. First it was done by sutures alone which have many disadvantages such as putting excessive strain on the surrounding tissue and also has high recurrence rate. Later on, a synthetic mesh as prolene mesh were used to provide better results and decrease complication and recurrence rates, however many methods were used to apply the mesh on the defect either onlay, sublay, inlay orpro-peritoneal approaches but still has disadvantages of recurrence which is low but still present, associated morbidity, wound complications and low patients' satisfaction [11].

During the past 10 years, laparoscopic repair of ventral hernia has become increasingly established in clinical practice [12], and aimed to be an acceptable and successful technique [13]. Laparoscopic hernia repair has many advantages as it can be done with relatively small incisions, repairing the hernia and placing the mesh with minimal injury to the surface of the abdomen, detection of occult hernia defects which are common in ventral hernia. The inspection during laparoscopy takes advantage of detecting occult hernia defects precisely, which result in an excellent outcome of laparoscopic ventral hernia repair [14].

Although laparoscopic ventral hernia repair has decreased complications compared to open techniques, it also has additional complications as port site hernia, intestinal and vessels injury. Recently single port access technique is applied to decrease these complications in spite of two port repair technique. There are many methods used in laparoscopic ventral hernia repair as IPOM [intra-peritoneal only mesh] just reduction the contents and tacks the mesh to abdominal wall without closure of the defect or closing the defect before application of mesh [13].

The aim of this study is to compare outcomes and results of closure versus non-closure of ventral hernia defect during laparoscopic ventral hernia repair.

\section{Patients and Methods}

This prospective randomized study had been conducted in Qena university hospitals, Qena faulty of medicine, South Valley University in co-operation with Aswan University Hospitals over a 36 months period between June 2015 and June 2018 on 60 patients with ventral hernia and they were subjected to laparoscopic ventral hernia repair with mesh. The study was approved by local ethical committee.

Patients were divided randomly into 2 groups: group 1 [non-closure group]: 30 patients with fixation of mesh without closing of the hernia defect, group 2 [closure group]: 30 patients with hernia defect closure before mesh fixation.

All patients presented to us in the outpatient clinic for elective operation [non 
complicated ventral hernia]. The clinical diagnosis of ventral hernia was based clinical examination [symptoms and signs]. All patients were evaluated preoperatively for fitness for surgery and anesthesia and treatment of any underlying causes of hernia or predisposing factors. Upon admission, patients were fully examined, routinely investigated and prepared for laparoscopic ventral hernia repair with mesh, all patients receive prophylactic antibiotics.

Inclusion criteria: All patient ages ranged from 18 to 60 years presenting with small and medium sized ventral hernias, patients were capable of understanding and giving written consent for laparoscopic treatment of ventral hernia.

Exclusion criteria: Large hernias, recurrent hernias, complicated ventral hernia as incarcerated, obstructed, strangulated, or infected hernias, cardiopulmonary diseases [for fear of the effect of $\mathrm{Co}_{2}$ pneumo-peritoneum and general anesthesia], severe liver disease, patients refuse to be operated by laparoscopic procedure or refuse to sign consent.

Preoperative preparation: Patients were fasting 6 hours before operation and shaving of abdominal hair and giving prophylactic antibiotics one hour before the operation. Patients were asked to void urine just before the operation and another one gram of third generation cephalosporin was given just at start of operation.

Anesthesia: Laparoscopic ventral hernia repair is usually performed under general endotracheal anesthesia with muscle relaxation.

Surgical techniques:

Group 1: Non-closure group: The patient is placed in the supine position with both arms tucked. If the hernia is in the midline, the surgeon can stand on either side of the patient, with the monitor directly opposite. If the hernia extends significantly to one side, initial trocar placement is done in the opposite side. Initially, the assistant stands on the same side as the surgeon, however, he may later have to move to the opposite side to help with dissection and stapling. A second monitor on the opposite side of the table is useful. After adequate general anesthesia was obtained, the abdomen was prepared and draped in the usual sterile manner. Pneumo-peritoneum was established by veress needle when possible, or an optical trocar allowing view of the abdominal wall layers during penetration. The position of the trocars varied depending on the size, location and number of existing hernia defects. In general, two $5-\mathrm{mm}$ and one $12-\mathrm{mm}$ trocars were placed along the left lateral abdomen.

The whole anterior abdominal wall must be completely free from adhesions, especially when there are multiple incisions and the hernia contents reduced. Adhesions near the bowel should be freed with scissors without any energy source to prevent thermal injury. Adhesions between the greater omentum and the abdominal wall may be severed with diathermy or ultrasonic harmonic Scalpel [Ethicon USA]. Clearance of regions covered with fatty tissue such as the falciform ligament to provide secure fixation of the mesh and not to miss any fascial defects. The peritoneal sac is left in situ. After completion of the dissection, 
the hernia defect was measured, and a mesh chosen to overlap all margins of the defect by at least $3-5 \mathrm{~cm}$ [Parietex TM Composite ventral patch, Covidien Ltd., UK]. The periphery of the hernia defect is evaluated by direct vision and palpation and is marked on the abdominal wall skin with a marker. The carbon dioxide should be released prior to measurement, revealing the true size of the hernia defect. The cranio-caudal and lateral measurements are taken to define the size of the mesh. The surgeon should add $5 \mathrm{~cm}$ to these measurements in both directions, which provides a 3-cm overlap of the edges of the hernia by the mesh.

After selection of the appropriate-sized mesh, 4 stay sutures were placed at four corners of the mesh and retrieved individually with a suture passer to provide fascial fixation of the mesh. The suture sites are numbered with a marker to allow easier orientation of the prosthetic mesh in the abdominal cavity. The tailored mesh is rolled tightly and inserted in the peritoneal cavity through the 12 mm trocar.

Then we unrolled mesh inside the abdomen and spread it under the defect. Two-mm skin incisions are made in the marked points on the abdominal wall. With the help of a fascial closure instrument [was inserted through each skin incision into the peritoneal cavity], the 2 ends of each suture are grasped and drawn outside through the skin incision by separate passages and at different angles. The suture ends are tied down extra-corporeally and buried subcutaneously. The tacks are placed at the margins of the mesh at $1-\mathrm{cm}$ intervals we used SECURESTRAP R [Ethicon, INC, USA], then the trocar were removed under direct vision and the fascia for any exposed $12-\mathrm{mm}$ port site was routinely closed with 0-Vicryl suture. Skin incisions were closed in subcuticular sutures.

Group 2: Closure group: The procedure was similar to the technique described above for non-closure. The only difference was that we performed a primary approximation of the fascial edges of the hernia defect with prolene sutures prior to mesh placement using intra-corporeal sutures using a needle and standard laparoscopic needle holders; Figures 1-3.

The following data were collected for each patient: age, sex, body mass index [BMI], previous hernia repairs, size and location of the fascial defect, operative time, estimated blood loss, co-morbidities, and length of hospital stay, complications, conversion rate, recurrences.

Follow up: Patients were examined postoperatively at 1 week, one month, 3 months, 6 months, 1 year, 18 months, 2 years, and thereafter as clinically indicated. Follow-up was achieved by reviewing medical records of clinic visits, and phone interview, WhatsApp chat.

We used Assessment of pain by visual analogue scale [VAS], the VAS from no pain [0] to worst pain imaginable [10].

Statistical analysis: the statistical analysis of data was done by using SPSS [Statistical Package for the Social Sciences ver. 10.0, SPSS Inc., Chicago, Illinois, USA] computer software for Statistical analysis under Microsoft window 8. For all statistical analyses, $\mathrm{p}>0.05$ was considered significant. 


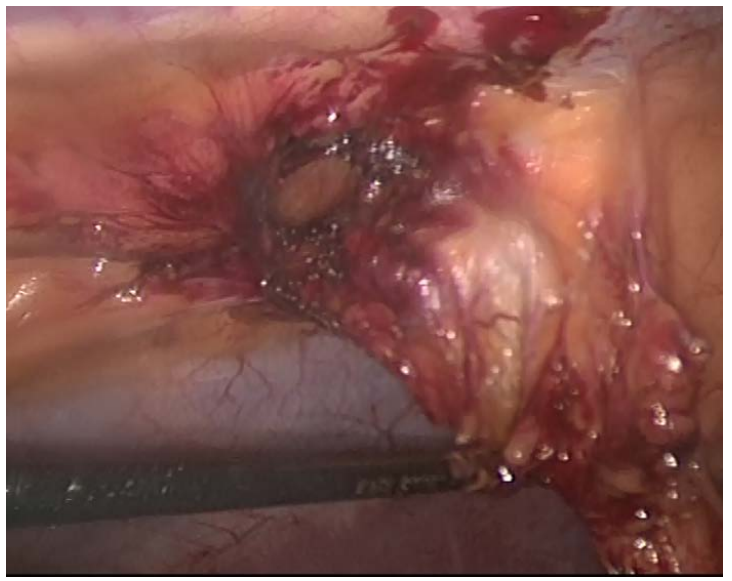

Figure 1. Reduction of content of ventral hernia and clear the adhesions between it and abdominal wall.

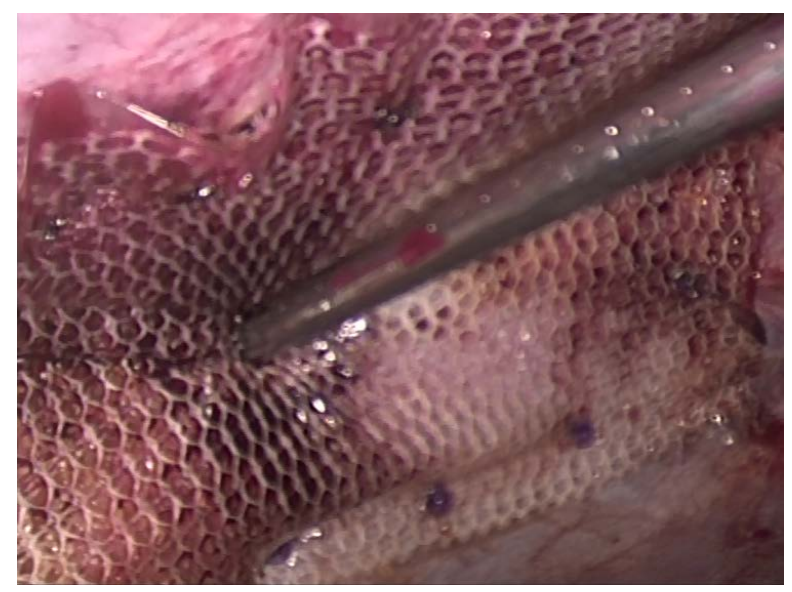

Figure 2. Fixation of mesh with tacker.

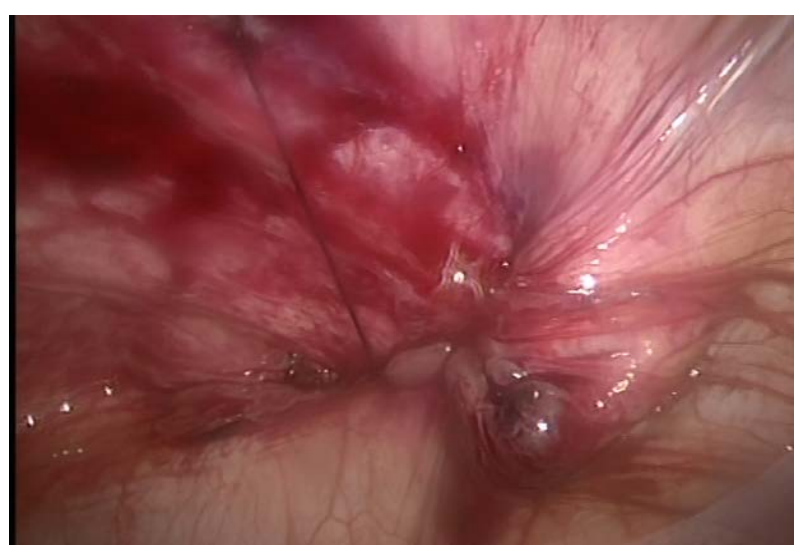

Figure 3. Closing of the defect of ventral hernia.

\section{Results}

60 patients with ventral abdominal hernia were included in this study between June 2015 and June 2018, all selected cases underwent laparoscopic ventral repair without conversion any of them. 60 patients were randomized classified in 
two groups, each group included 30 patients: group 1 treated with laparoscopic reduction of contents and fixation of mesh without closing the hernia defect [non-closure group] and group 2 treated with laparoscopic reduction of content and closing of the defect and fixation of mesh.

As regarding patient's parameters: There were 30 patients in the non-closure group [group 1] most of them were females [20 patients with parentage $66.67 \%$ and males were only 10 patients $33.33 \%$ ] with mean age was 33 years [ranges from 18 - 55 years] but in group 2 defect closure group, there were 30 patients 18 were females and 12 were males with mean age were 35 [ranges from 20 - 60 years]. Mean BMI were 33 in group 1 and 29 in group 2 with ranges varies from 22 - 40. Sizes of defect by ultra-sonography were varies in group 1 from 0.5 to 3 $\mathrm{cm}$ with mean $2 \mathrm{~cm}$ and in group 2 varies from $1-4 \mathrm{~cm}$ with mean $2.5 \mathrm{~cm}$ (Table 1).

Operatives and post-operative outcomes: The operative time for group 1 [Non closure group] ranged between 50 - 90 minutes with a mean time 75 minutes. The operative time for group 2 [closure group] ranged between [80 - 130] minutes with a mean time 110 minutes. As regarding the blood loss, there is no significant difference in blood loss in both groups.

As regarding intra-operative complications, iatrogenic injury of the small intestine was occurred accidentally in one patient during dissection and was repaired by simple stitches in group 2 and small intestinal serosal tear in one patient in group 1 , and serosa was approximated by $3 / 0$ vicryl suture but these accidents hadn't any relation with types of closure and procedure was completed laparoscopically. Post-operative hospital stay ranged from $24-72$ hours with a mean of 40 hours; only one patient had been stayed in hospital for 4 days due to iatrogenic small intestinal injury. Most of patients discharged after 2 days and oral feeding started after 6 hours post operative for most of patients with early mobilization just after being fully conscious and return to their daily activities within 2 days and return to work after one week [within 10 days].

In group 1, 5 patients suffer from postoperative mild pain [33\%] and take single dose of injected analgesic, 7 patients suffer from moderate pain [47\%] which response to double dose of injected analgesic, 3 patients suffer from severe pain [20\%]. One patient experienced chronic and neuropathic pain. But in group 2, 3 patients suffer from mild pain [20\%] and take single dose of injected analgesic, 5 patients suffer from moderate pain [33\%] which response to double dose

Table 1. Patient's parameters.

\begin{tabular}{ccc}
\hline Parameters & Group 1 & Group 2 \\
\hline Number of patients & 30 & 30 \\
M:F & $2: 1$ & $1.5: 1$ \\
Mean age & 33 & 35 \\
BMI & 33 & 29 \\
Size of the defect & $2 \mathrm{~cm}$ & $2.5 \mathrm{~cm}$ \\
\hline
\end{tabular}


of injected analgesic, 7 patients suffer from severe pain [47\%]. 3 patients [33\%] experienced chronic and neuropathic pain. Seroma had been occurred in $17 \mathrm{pa}-$ tients [56.7\%] in group 1 and only in 5 patients in group 2.

Recurrence occurred in two patients [6.67\%] in group 1 and appeared after 6 months but no recurrence had been detected in group 2 during follow up period. Ileus was developed in one patient [3.34\%] in group 1 and 2 patients [6.67\%] in group 2 and treated with conservative treatment with IV fluids and NGT [nasogastric tube] and improved later on within 24 hours. The bulging appears postoperative in 20 patients [66.6\%] in group 1 and in 5 patients [16.6\%] in group 2. Wound infection had occurred in one patient in both groups [6.6\%] (Table 2).

\section{Discussions}

Ventral hernias can be defined as defects of the anterior abdominal wall and it is classified into congenital [epigastric, umbilical and Spigelian] and acquired [incisional] [1] [15]. Incisional hernia is a common complication of laparotomies with incidence ranges between $2 \%$ and 13\% [1] [15] [15].

In the last decades, laparoscopy has made significant strides and has become the gold standard procedure in many surgical fields. Laparoscopic ventral hernia repair was started early at the beginning of the 1990 and was described on firsts by Leblanc in 1993 for all types of hernia [5] [17]. From this time, laparoscopic ventral hernia repair was controversial, because various studies reported an early recurrence rate as high as $25 \%$ [18]. But now, after decades of experience in

Table 2. Operative and post operative outcomes.

\begin{tabular}{|c|c|c|c|}
\hline Parameter & Group 1 & Group 2 & $P$ value \\
\hline Operative time & 50 - 90 Ms. & 80 - 130 Ms. & \\
\hline Mean operative time & 75 Ms. & $110 \mathrm{Ms}$. & 0.021 \\
\hline Blood loss & $20-30 \mathrm{ml}$ & $25-40 \mathrm{ml}$ & 0.243 \\
\hline Postoperative hospital stay & $24-72$ hours & $24-72$ hours & 0.543 \\
\hline $\begin{array}{l}\text { Postoperative pain score } \\
\text { [mean] }\end{array}$ & 2 & 2 & \\
\hline Return to daily activities & Within 2 days & Within 2 days & 0.1 \\
\hline Return to work & $\begin{array}{l}7 \text { - } 12 \text { days with } \\
\text { mean } 10 \text { days }\end{array}$ & $\begin{array}{l}8 \text { - } 14 \text { days with } \\
\text { mean } 12 \text { days }\end{array}$ & 0.543 \\
\hline Recurrence & 4 [13.33\%] & $1[3.33 \%]$ & 0.011 \\
\hline Ileus & $1[3.33 \%]$ & $2[6.67 \%]$ & 0.657 \\
\hline Seroma & $17[65.675]$ & $5[16.16]$ & 0.0345 \\
\hline Wound infection & 1 & 1 & 0.765 \\
\hline Port site hernia & - & - & \\
\hline appearance of bulge & 20 & 5 & 0.002 \\
\hline $\begin{array}{l}\text { Postoperative neuropathy } \\
\text { [chronic pain] }\end{array}$ & 1 & 3 & 0.0678 \\
\hline
\end{tabular}


laparoscopic hernia surgery, laparoscopic ventral hernia repair has gained worldwide acceptance and has became the first choice for ventral hernia repair in many laparoscopic centers. So during the past 10 years, laparoscopic repair of ventral hernia has become increasingly established in clinical practice [1] and the surgical technique has improved and has proven to be an effective treatment option [2] [19]. Now, it has become a standard approach to repair many types of ventral and incisional hernias [19]. It has been shown to be superior to open hernia repair, with generally fewer complications and recurrences [20]. It is minimally invasive repair even more so appropriate in the morbidly obese patients [21], and it is more beneficial in patient with a minimum defect of $3 \mathrm{~cm}$, additionally, closing the defect primarily has been advocated. However, primary fascial closure during laparoscopic hernia repair has not been proven to decrease complications when compared with bridged techniques [22] [23].

There are many techniques for laparoscopic hernia repair as IPOM just fixation of mesh after reduction of content without closing the defect of hernia or closing the defect either extracorporeal or intra-corporeal or using END-STITCH suturing device. In study by, Franklin et al. [24] published in 2004, they reported their 11 years experience with laparoscopic ventral hernia repair and their technique included primary closure of the defect before mesh placement. And he reported that closure of defect had many benefits including lower recurrence rate $[2.9 \%]$ and fewer complications, a lower wound and mesh infection rate [10.1\%] at a mean follow-up of 47.1 months. Since then, different defect closure techniques have been described, and all have advantages and disadvantages [24].

Laparoscopic ventral hernia repair technique without defect closure has many advantages as it is simple easy to learn, not need extra-experience in laparoscopic intra-corporal suturing, time saving, but also have many disadvantages were reported as bulging phenomenon as the mesh bulges through the defect. Also there is another disadvantage in which mesh can becomes in contact with the skin which may result in fistula formation beside high recurrence rats especially in larger defect. Conversely, there are many advantages and benefits with closing the defect of ventral hernia, as it is approved that by closing the defect, the repair is stronger and more reliable, also it has been suggested that by approximating the fascial edges, a more physiologic restoration of abdominal wall function is achieved. When the defect is closed, the mesh is never in contact with the skin because the abdominal wall muscle and fascia provide a physical barrier. This may also help prevent mesh erosion of the skin and subsequent infections [25] [26], but also primary closure of the hernial defect is, technically complex, as shown by previous experience [27] [28] [29].

As regard recurrences after laparoscopic ventral hernia repair are variables in many studies but generally are range from $4.2 \%$ to $16.7 \%$ and some authors reported lower recurrence rates with defect closure from $0 \%$ to $2.9 \%$. In this study, 30 patients with a laparoscopic ventral hernia defect closure of the defect with mesh fixation, only in one patient had recurrent during a mean follow-up of 12 months [this low recurrence rate may also attributed to selection criteria of cases 
as we select small and medium sized and denovo ventral hernias] but in the non-defect closure group, we found that the recurrence rates were higher [in 4 patients with percentage was $13.33 \%$ ]. In study by Roberto Rea et al. on 43 patients, only 2 patients had recurrences and just small defect by ultra-sonography [30].

Some authors have also thought that closing the defect with percutaneous sutures were associated with abdominal discomfort [up to 6 months after surgery], pain and neuralgia but this may be due to fixation techniques, whether tacks, sutures or a combination other than closing the defect.

The method of mesh fixation is important as it is found in a meta-analysis study that when tackers are used alone, the operative time was shorter with less postoperative pain. In our study we used a both of tacks and sutures. Four corners of the mesh were secured with trans-fascial sutures and then tacks were applied.

Regarding seroma formation, there are a significant difference between two groups as it was in group 1 without defect closure which more higher than that in group 2 with defect closure [65.67\% vs $16.16 \%$ ] and this may due to that fluid collecting in the sac was difficult to drain back into the peritoneal cavity and this result was similar to result that reported by Franklin et al. [24], who reported rates of $15 \%-20 \%$ for seroma formation in defect closure.

As regarding operative time, it was ranged from 50 - 90 minutes with median 75 minutes in group 1 and was ranged from 80 - 130 in group 2 with median 110 minutes and the operative time was prolonged when using trans-fascial or intra-corporeal suturing.

Our results that were obtained from this study demonstrate that the laparoscopic ventral hernia repair with defect closure is safe and feasible with lower recurrence rates and low seroma rates and fewer wound complication. Also operative time to somewhat may be prolonged but with time and with more experience it will be shorter.

In general we can concluded that the use of laparoscopic techniques for hernia repair with defect closure has lower recurrence, infection, seroma rates and decreased the length of hospital stay, however laparoscopy application is limited by the procedures that are amenable to its adaptation and the outcomes. Many hernia repairs cannot be undertaken laparoscopically because of extensive adhesions, large hernia defects, and the level of contamination. Size of the hernia defect plays a main role in the technique that we will use. For examples small defect closure may not be needed unless a single defect is large [ $3 \mathrm{~cm}$ wide]. Closure of a larger wide defect can be challenging. The largest defect reported being closed was $12 \mathrm{~cm}$ wide. Although there is no accepted way to evaluate abdominal compliance and elasticity in the clinical setting; easily distendable abdominal walls are more amenable to primary fascial closure [26].

\section{Conclusion}

Laparoscopic ventral hernia repair is safe and feasible, although laparoscopic 
ventral hernia repair without closure of the defect is easy with less operative time and does not need extra-experience in intra-corporeal suturing but its benefit was in smaller defect $[<3 \mathrm{~cm}]$ and larger defect need a laparoscopic ventral hernia repair with defect closure as it provides durable repair with low recurrence rates, lower seroma, lower wound infection with good cosmetic result, with good patients satisfactions, but still there is a slightly higher incidence of seroma formation in both groups and especially in non-closure group and this needs more studies or modification of these techniques to reduce seroma and bulge phenomenon. Also, the short follow-up period and to somewhat a small number of patients included in this study do not allow for a safe conclusions regarding long-term results and recurrences rates so we expect similar or better results with our techniques in larger studies.

\section{Ethical Consideration}

This study was approved by local ethics committee.

\section{Conflicts of Interest}

Authors declare no conflict of interest.

\section{References}

[1] Banerjee, A., Beck, C., Narula, V.K., Linn, J., Noria, S., Zago, L.B. and Mikami, D.J. (2012) Laparoscopic Ventral Hernia Repair: Does Primary Repair in Addition to Placement of Mesh Decrease Recurrence? Surgical Endoscopy, 26, 1264-1268. https://doi.org/10.1007/s00464-011-2024-3

[2] Lomanto, D., Iyer, S.G., Shabbir, A. and Cheah, W.K. (2006) Laparoscopic versus Open Ventral Hernia Mesh Repair: A Prospective Study. Surgical Endoscopy, 20, 1030-1035. https://doi.org/10.1007/s00464-005-0554-2

[3] Ahmed, D. and Khan, M.J. (1995) Use of Mesh in the Management of Recurrent Incisional Hernias. Pakistan Journal of Surgery, 11, 101-102.

[4] Stoppa, R.E. (1989) The Treatment of Complicated Groin and Incisional Hernias. World Journal of Surgery, 13, 545-554. https://doi.org/10.1007/BF01658869

[5] Hoer, J., Lawong, G., Klinge, U. and Schumpelick, V. (2002) Factors Influencing the Development of Incisional Hernia. A Retrospective Study of 2983 Laparotomy Patients over a Period of 10 Years. Chirurg, 73, 474-480.

[6] Knuutinen, A., Kokkonen, N., Risteli, J., et al. (2002) Smoking Affects Collagen Synthesis and Extracellular Matrix Turnover in Human Skin. British Journal of Dermatology, 146, 588-594. https://doi.org/10.1046/j.1365-2133.2002.04694.x

[7] Sorensen, LT. (2006) Effect of Life Style, Gender and Age on Collagen Formation and Degradation. Hernia, 10, 456-461. https://doi.org/10.1007/s10029-006-0143-X

[8] Van't Riet, M., Steyerberg, E.W., Nellensteyn, J., Boryer, H.G. and Jeekel, J. (2002) Meta-Analysis of Techniques for Closure of Midline Abdominal Incisions. British Journal of Surgery, 80, 1350-1356. https://doi.org/10.1046/j.1365-2168.2002.02258.x

[9] Hernias, A.J. (2001) Maingot's Abdominal Operations. 10th Edition, Appleton \& Lange, Connecticut, 479-580.

[10] Wechsler, R.G., Kurtz, A.B., Needleman, L., et al. (1995) Cross-Sectional Imaging of 
Abdominal Wall Hernias. $A J R, 135,517-521$.

[11] Stylopoulos, N. and Rattner, D. (2005) The History of Hiatal Hernia Surgery from Bowditch to Laparoscopy. Annals of Surgery, 15, 185-193.

[12] Gut, C., Qniu, T., Mehrabi, A. and Buchler, W. (2004) Fewer Adhesions Induced by Laparoscopic Surgery. Surgical Endoscopy, 18, 898-906. https://doi.org/10.1007/s00464-003-9233-3

[13] Moulhas, A., Podalsky, E., Curcello, P., et al. (2009) Single Port Access Ventral Hernia Repair. Surgical Endoscopy, 23, S094.

[14] Indani, H., Sasaki, H., Yashioka, T., Asami, S., Kurose, Y., Nojima, H., Muro, M., Yamashita, T., Hirata, M., Kumano, K. and Kin, H. (2009) Laparoscopic Ventral Hernia Repair Hasan Advantage of Detecting Occult Hernia Defects. Surgical Endoscopy, 23, 454 .

[15] Seymour, N.E. and Bell, R.L. (2010) Abdominal Wall, Omentum, Mesentery, and Retroperitoneum. In: Schwartz's Principles of Surgery, 9th Edition, Chapter 35, McGraw-Hill, New York.

[16] Santora, T.A. and Roslyn, J.J. (1993) Incisional Hernia. Surgical Clinics of North America, 73, 557-570. https://doi.org/10.1007/s00464-011-2024-3

[17] Leblanc, K.A. and Booth, W.V. (1993) Laparoscopic Repair of Incisional Abdominal Hernias Using Expanded Polytetrafluororthylene: Preliminary Findings. Surgical Laparoscopy \& Endoscopy, 3, 39-41.

[18] Wintringer, A. (2006) Total Extraperitoneal Hernia Repair after Radical Prostatectomy or Previous Abdominal Surgery. Surgical Endoscopy, 20, 473-476.

[19] Dapri, G., Bruyns, J., Paesmans, M., et al. (2013) Single-Access Laparoscopic Primary and Incisional Prosthetic Hernia Repair: First 50 Patient. Hernia, 17, 619-626. https://doi.org/10.1007/s10029-012-1025-Z

[20] Cuccurullo, D., Piccoli, M., Melotti, G., et al. (2013) Laparoscopic Ventral Incisional Hernia Repair: Evidence-Based Guidelines of the First Italian Consensus Conference. Hernia, 17, 557-566.

[21] Raftopoulos, I. and Courcoulas, A.P. (2007) Outcome of Laparoscopic Ventral Hernia Repair in Morbidly Obese Patients with a Body Mass Indexes Exceeding 35 $\mathrm{kg} / \mathrm{m}^{2}$. Surgical Endoscopy, 21, 2293-2297.

[22] Wennergren, J.E., Askenasy, E.P., Greenberg, J.A., et al. (2015) Laparoscopic Ventralhernia Repair with Primary Fascial Closure versus Bridged Repair: A Risk-Adjusted Comparative Study. Surgical Endoscopy.

[23] Zhang, Y., Zhou, H., Chai, Y., et al. (2014) Laparoscopic versus Open Incisional Andventral Hernia Repair: A Systematic Review and Meta-Analysis. World Journal of Surgery, 38, 2233-2240. http://www.appliedmedical.com/Products/GelPoint

[24] Franklin, M.E., Gonzalez, J.J., Glass, J.L. and Manjarrez, A. (2004) Laparoscopic Ventral and Incisional Hernia Repair: 11-Year Experience. Hernia, 8, 23-27. https://doi.org/10.1007/s10029-003-0163-8

[25] Agarwal, B.B., Agarwal, S. and Mahajan, K.C. (2008) Laparoscopicventral Hernia Repair: Innovative Anatomical Closure, Mesh Insertion without $10 \mathrm{~mm}$ Transmyofascial Port, and a Traumatic Mesh Fixation: A Preliminary Experience of a New Technique. Surgical Endoscopy, 23, 900-905.

[26] Chelala, E., Thoma, M., Tatete, B., Lemye, A.C., Dessily, M. and Alle, J.L. (2007) The Suturing Concept for Laparoscopic Mesh Fixation in Ventral and Incisional Hernia Repair: MID Term Analysis of 400 Cases. Surgical Endoscopy, 21, 391-395. https://doi.org/10.1007/s00464-006-9014-x 
[27] Orenstein, S.B., Dumeer, J.L., Monteagudo, J., Poi, M.J. and Novitsky, Y.W. (2011) Outcomes of Laparoscopic Ventral Hernia Repair with Routine Defect Closure Using "Shoelacing" Technique. Surgical Endoscopy, 25, 1452-1457. https://doi.org/10.1007/s00464-010-1413-3

[28] Melvin, W.S. and Renton, D. (2011) Laparoscopic Ventral Hernia Repair. World Journal of Surgery, 35, 1496-1499. https://doi.org/10.1007/s00268-011-1028-4

[29] Elazary, R., Abu-Gazala, M., Schlager, A., Khalaileh, A., Shussman, N., Rivkind, A.I. and Mintz, Y. (2011) Trans-Fascial Laparoscopic Mesh Fixation: A Procedural Comparison Using the Standard Suture Passer versus Imesh Stitcher ${ }^{\text {TM }}$ Device. Hernia, 15, 321-324. https://doi.org/10.1007/s10029-011-0789-x

[30] Rea, R., Falco, P., Izzo, D., Leongito, M. and Amato, B. (2012) Laparoscopic Ventral Hernia Repair with Primary Transparietal Closure of the Hernial Defect Rea et al. BMC Surgery, 12, S33.

http://www.biomedcentral.com/1471-2482/12/S1/S33 